\title{
ON PARABOLIC PROBLEMS WITH NON-LIPSCHITZ NONLINEARITY
}

\author{
OLEG ZUBELEVICH \\ DEPARTMENT OF DIFFERENTIAL EQUATIONS AND MATHEMATICAL PHYSICS \\ PEOPLES FRIENDSHIP UNIVERSITY OF RUSSIA \\ ORDZHONIKIDZE ST., 3, 117198, MOSCOW, RUSSIA \\ E-MAIL : OZUBEL@YANDEX.RU
}

\begin{abstract}
We consider parabolic problems with non-Lipschitz nonlinearity in the different scales of Banach spaces and prove local-in-time existence theorem. New class of parabolic equations that have analytic solutions is obtained.
\end{abstract}

\section{INTRODUCTION}

This paper is devoted to quasi-linear parabolic equations with a nonLipschitz nonlinearity. In the classical setup a quasi-linear initial value parabolic problem has the form

$$
u_{t}=f\left(t, u, \nabla^{k} u\right)+A u,\left.\quad u\right|_{t=0}=\hat{u} .
$$

Here $A$ is a linear elliptic operator of order $n$ and the term $\nabla^{k} u$ symbolizes the derivatives of $u$ up to order $k$. Besides this, equation (1.1) must be provided with the boundary conditions.

If the function $\hat{u}$ belongs to a suitable space, the mapping $f$ is Lipschitz in a certain sense and $k<n$ then problem (1.1) has a unique local-intime solution. This simple observation easily follows from the contracting mapping principle.

We consider the case when the function $f$ is non-Lipschitz. It is well known that in general situation, in infinitely dimensional Banach space, an initial value problem for differential equation with non-Lipschitz right hand side does not have solutions $[4,12,5]$. Nevertheless, as a rule, the initial value problem lives not in a single Banach space but in a scale of Banach spaces and in addition this scale is completely continuous embedded. Such scales for example are the scale of Sobolev spaces, the scale of analytic

2000 Mathematics Subject Classification. 35K90, 35R05, 35R10.

Key words and phrases. Peano theorem, abstract Cauchy problem, nonlocal problems, functional-differential equations, integro-differential equations, quasilinear parabolic equations.

Partially supported by grants RFBR 02-01-00400. 
functions. This observation prompts that to find a solution one should study the problem in the whole scale.

Let us note another feature of equations (1.1). If we reject the Lipschitz hypothesis on $f$ then we obtain a class of systems that have existence theorem even in the case when $k \geq n$. Such type systems remain parabolic in some certain generalized sense.

This effect takes place not only for parabolic equations. If we consider the Cauchy-Kowalewski problem in the non-Lipschitz setup [13] then there are equations such that the order of derivatives in the right side is greater than in the left one but the solution exists.

These problems do not belong to the classical partial differential equations but to the functional-differential equations and the differential equations with nonlocal terms.

The main mathematical tool we use is a locally convex space version of the Schauder fixed point theorem and theory of scales of Banach spaces. Another approaches to the abstract parabolic problems in the Lipschitz setup contain in [1], [3].

\section{MAIN THEOREM}

Consider two scales of Banach spaces $\left\{E_{s},\|\cdot\|_{s}^{E}\right\}_{s>0}$ and $\left\{G_{s},\|\cdot\|_{s}^{G}\right\}_{s>0}$ such that $E_{s} \subseteq G_{s}$ for all $s>0$. All the embeddings $E_{s+\delta} \subseteq E_{s}, \quad \delta>0$ are completely continuous and

$$
\|\cdot\|_{s}^{E} \leq\|\cdot\|_{s+\delta}^{E}
$$

The parameter $s$ may not necessarily be ran through all the positive real numbers. We do not use the spaces $E_{s}, G_{s}$ with big $s$ and one can assume for example that $s \in(0,1)$. It is just for simplicity's sake that we consider $s>0$.

Introduce constants $C, T, R>0, \quad \phi, \alpha \geq 0$.

Let $S^{t}: G_{s} \rightarrow E_{s}, \quad t>0$ be a strongly continuous linear semigroup in the following sense. For any $u \in E_{s}$ one has

$$
\left\|S^{t} u-u\right\|_{s}^{E} \rightarrow 0 \quad \text { as } \quad t \searrow 0 \quad \text { and } \quad\left\|S^{t} u\right\|_{s}^{E} \leq C\|u\|_{s}^{E} .
$$

Definition 1. The semigroup $S^{t}$ is said to be parabolic if there exists a constant $\gamma>1$ such that for any $\delta, t>0, \quad \delta^{\gamma}<t<T$ we have

$$
\left\|S^{t} u\right\|_{s+\delta}^{E} \leq \frac{C}{t^{\phi}}\|u\|_{s}^{G}
$$

Let $B_{s}(r)$ be an open ball of the space $E_{s}$ with radius $r$ and center at the origin. Suppose a function $f:(0, T] \times \bar{B}_{s+\delta}(R) \rightarrow G_{s}$ to be continuous and such that if $(s+\delta)^{\gamma}<t \leq T$ and $u \in \bar{B}_{s+\delta}(R)$ then the following inequality holds

$$
\|f(t, u)\|_{s}^{G} \leq \frac{C}{\delta^{\alpha}}
$$


Remark 1. A case when

$$
\|f(t, u)\|_{s}^{G} \leq \frac{C}{t^{\beta} \delta^{\alpha}}, \quad \beta>0
$$

is rather usual but since $\delta^{\gamma}<t$ this case reduces to (2.3): $C /\left(t^{\beta} \delta^{\alpha}\right) \leq$ $C / \delta^{\beta \gamma+\alpha}$.

We proceed with two setups of our problem. The first one is a classical setup and we find classical solutions and the second one is a generalized setup to obtain generalized solutions.

In the generalized setup we are looking for solutions to the following integral equation

$$
u(t)=\int_{0}^{t} S^{(t-\xi)} f(\xi, u(\xi)) d \xi .
$$

In the classical setup we make several additional assumptions. Namely, suppose that $G_{s}=E_{s}$. Introduce a linear operator $A: E_{s+\delta} \rightarrow E_{s}$ and assume that the semigroup $S^{t}$ is generated by this operator: $S^{t}=e^{A t}$ such that for any $u \in E_{s+\delta}$ we have

$$
\lim _{h \rightarrow 0+}\left\|\frac{1}{h}\left(e^{A h}-\operatorname{id}_{E_{s+\delta}}\right) u-A u\right\|_{s}^{E}=0 .
$$

In the classical setup our problem has the form

$$
\begin{aligned}
u_{t} & =f(t, u)+A u, \\
\left.u\right|_{t=0} & =0 .
\end{aligned}
$$

The sense of initial condition (2.7) will be clear in the sequel.

Now we give a definition.

Definition 2. We shall say that problem (2.6) or (2.4) is parabolic if the semigroup $S^{t}$ is parabolic and

$$
\chi=\phi+\frac{\alpha}{\gamma}<1
$$

In case of remark $1 \chi=\phi+\beta+\alpha / \gamma$.

Let a space $E^{1}(T), \quad T>0$ be given by the formula

$$
E^{1}(T)=\bigcap_{0<s^{\gamma}<\tau<T} C^{1}\left((\tau, T), E_{s}\right) .
$$

This space consists of all functions $u$ that map any number $t \in(0, T)$ to the element $u(t) \in \bigcap_{0<s^{\gamma}<t} E_{s}$ and the restriction $\left.u\right|_{(\tau, T)}$ belongs to the space $C^{1}\left((\tau, T), E_{s}\right)$ for all $s \in\left(0, \tau^{1 / \gamma}\right)$.

Theorem 1. 1) Classical setup. Suppose that problem (2.6) is parabolic. Then there exists a constant $T_{*}>0$ such that this problem has a solution $u(t) \in E^{1}\left(T_{*}\right)$, and for any constant $c \in(0,1)$ one has

$$
\|u(t)\|_{c t^{1 / \gamma}}^{E} \rightarrow 0 \quad \text { as } \quad t \searrow 0
$$

The function $u(t)$ also solves equation (2.4). 
2) Generalized setup. Suppose that problem (2.4) is parabolic. Then there exists a constant $T_{*}>0$ such that this problem has a solution

$$
u(t) \in E\left(T_{*}\right)=\bigcap_{0<s^{\gamma}<\tau<T_{*}} C\left(\left(\tau, T_{*}\right), E_{s}\right) .
$$

In both cases the constants $T_{*}$ depends only on $C, \alpha, \gamma, \phi$.

The proof of theorem 1 contains in sections 3,4 .

Then to illustrate the effect discussed in the Introduction, theorem 1 is applied to a nonlocal parabolic problem. To compare our result with the known one we also consider the Navier-Stokes equation.

If $A$ is the classical Laplace operator and the parabolic equation is considered in a suitable domain then $\gamma=2$ and the inequality from formula (2.8) takes the form $0<s^{2}<\tau$.

The parameter $s$ symbolizes a spatial variable, so that this inequality specifies the parabolic domain in the plane $(\tau, s)$. This endows the term "parabolic equation" with the new sense.

Let us remark that if $G_{s}=E_{s}=\mathbb{R}^{m}, \quad\|\cdot\|_{s}^{E}=|\cdot|, \quad s>0$ and $A=0$ then theorem 1 generalizes classical Peano's theorem to the case when the right side of the equation satisfies $(2.3)$ with $s=\delta=(t / 3)^{1 / \gamma}$.

\section{Preliminaries on FunCtional analysis}

In this section we collect several facts from functional analysis. These facts will be useful in the section 4 when we prove theorem 1 .

Consider the spaces

$$
C\left([\tau, T], E_{\mu \tau^{1 / \gamma}}\right), \quad 0<\mu<1, \quad 0<\tau<T
$$

with standard norms. Now we construct the projective limit of these spaces. Define a space $E(T)$ as follows

$$
E(T)=\bigcap_{0<\mu<1} \bigcap_{0<\tau<T} C\left([\tau, T], E_{\mu \tau^{1 / \gamma}}\right) .
$$

There is another equivalent definition of the space $E(T)$ :

$$
E(T)=\bigcap_{0<s^{\gamma}<\tau<T} C\left([\tau, T], E_{s}\right) .
$$

Being endowed with a collection of seminorms

$$
\|u\|_{\tau, \mu}=\max _{\tau \leq \xi \leq T}\|u(\xi)\|_{\mu \tau^{1 / \gamma}}^{E}, \quad u \in E(T)
$$

the space $E(T)$ becomes a locally convex topological space.

These seminorms obviously satisfy the following inequalities

$$
\begin{aligned}
\|u\|_{\tau, \mu} & \leq\|u\|_{\tau, \mu+\delta}, \quad \delta>0, \\
\|u\|_{\tau, r \mu} & \leq\|u\|_{r^{\gamma} \tau, \mu}, \quad 0<r \leq 1 .
\end{aligned}
$$


Indeed, formula (3.2) follows from (2.1) directly. Formula (3.3) is a result of the estimate

$$
\|u\|_{\tau, r \mu}=\max _{\tau \leq \xi \leq T}\|u(\xi)\|_{\mu\left(r^{\gamma} \tau\right)^{1 / \gamma}}^{E} \leq \max _{r^{\gamma} \tau \leq \xi \leq T}\|u(\xi)\|_{\mu\left(r^{\gamma} \tau\right)^{1 / \gamma}}^{E}=\|u\|_{r^{\gamma} \tau, \mu} .
$$

Formulas (3.2), (3.3) imply that the space $E(T)$ is first countable: the topology of this space can be defined by the seminorms (3.1) only with $\mu, \tau \in \mathbb{Q}$.

Recall the Arzela-Ascoli theorem [10]:

Theorem 2. Let $H \subset C([0, T], X)$ be a set in the space of continuous functions with values in a Banach space $X$. Assume that the set $H$ is closed, bounded, uniformly continuous and for every $t \in[0, T]$ the set $\{u(t) \in X\}$ is a compact set in the space $X$. Then the set $H$ is a compact set in the space $C([0, T], X)$.

Now we shall establish an analogue of this result.

Proposition 1. Suppose that a set $K \subset E(T)$ is closed. Then $K$ is a compact set if the following two conditions are fulfilled.

The set $K$ is bounded.

For any $\varepsilon>0$ and for any $\tau \in(0, T), \quad \mu \in(0,1)$ there exists a constant $\delta>0$ such that if $t^{\prime}, t^{\prime \prime} \in[\tau, T], \quad\left|t^{\prime}-t^{\prime \prime}\right|<\delta$ then

$$
\sup _{u \in K}\left\|u\left(t^{\prime}\right)-u\left(t^{\prime \prime}\right)\right\|_{\mu \tau^{1 / \gamma}}^{E}<\varepsilon .
$$

(This means that $K$ is a uniformly continuous set.)

First prove a lemma.

Lemma 1. Let $\left\{v_{j}\right\} \subseteq K$ be a sequence. Then for any $\tau \in(0, T)$ the sequence $\left\{v_{j}\right\}$ contains a subsequence that is convergent in all the norms $\|\cdot\|_{\tau, \mu}, \quad \mu \in(0,1)$.

Proof. Indeed, take an increasing sequence $\mu_{k} \rightarrow 1, \mu_{1}>0$ and fix any value of $\tau \in(0, T)$. Since the sequence $\left\{v_{j}\right\}$ is bounded and uniformly continuous in $C\left([\tau, T], E_{\mu_{2} \tau^{1 / \gamma}}\right)$ then by theorem 2 it contains a subsequence $\left\{v_{j}^{1}\right\}$ that is convergent in $C\left([\tau, T], E_{\mu_{1} \tau^{1 / \gamma}}\right)$.

Further since the sequence $\left\{v_{j}^{1}\right\}$ is bounded and uniformly continuous in $C\left([\tau, T], E_{\mu_{3} \tau^{1 / \gamma}}\right)$ one can pick a subsequence $\left\{v_{j}^{2}\right\} \subseteq\left\{v_{j}^{1}\right\}$ such that the sequence $\left\{v_{j}^{2}\right\}$ is convergent in $C\left([\tau, T], E_{\mu_{2} \tau^{1 / \gamma}}\right)$ etc.

By inequality (3.2) the diagonal sequence $\left\{v_{j}^{j}\right\}$ converges in all the norms $\|\cdot\|_{\tau, \mu}, \quad \mu \in(0,1)$ with this fixed $\tau$.

Proof of proposition 1. A set $P=\mathbb{Q} \bigcap(0, T)$ is countable. So we can number its elements as follows $P=\left\{\tau_{i}\right\}_{i \in \mathbb{N}}$.

We must show that any sequence $\left\{u_{j}\right\} \subseteq K$ contains a convergent subsequence $\left\{u_{j_{k}}\right\}$. 
By lemma 1 there is a subsequence $\left\{u_{j}^{1}\right\} \subseteq\left\{u_{j}\right\}$ that is convergent in all the norms $\|\cdot\|_{\tau_{1}, \mu} \quad \mu \in(0,1)$. By the same argument there is a subsequence $\left\{u_{j}^{2}\right\} \subseteq\left\{u_{j}^{1}\right\}$ that is convergent in all the norms $\|\cdot\|_{\tau_{2}, \mu} \quad \mu \in(0,1)$ etc.

The diagonal sequence $\left\{u_{j}^{j}\right\}$ is convergent in all the norms $\|\cdot\|_{\tau_{k}, \mu}, \quad k \in$ $\mathbb{N}, \quad \mu \in(0,1)$.

By inequality (3.3) the sequence $\left\{u_{j}^{j}\right\}$ is convergent in all the norms $\|$. $\|_{\tau, \mu}, \quad \tau \in(0, T), \quad \mu \in(0,1)$.

Proposition 1 is proved.

Lemma 2. Let $X, Y$ be Banach spaces. Suppose that $A_{a}: X \rightarrow Y, \quad a^{\prime}>$ $a>0$ is a collection of bounded linear operators such that for each $x \in X$ we have

$$
\sup _{a^{\prime}>a>0}\left\|A_{a} x\right\|_{Y}<\infty, \quad\left\|A_{a} x\right\|_{Y} \rightarrow 0 \quad \text { as } \quad a \rightarrow 0 .
$$

Then for any compact set $B \subset X$ it follows that

$$
\sup _{x \in B}\left\|A_{a} x\right\|_{Y} \rightarrow 0 \quad \text { as } \quad a \rightarrow 0
$$

This result is a direct consequence of the Banach-Steinhaus theorem [10]. Let us recall a generalized version of the Schauder fixed point theorem.

Theorem 3 ([2]). Let $W$ be a closed convex subset of the locally convex space $E$. Then a compact continuous mapping $f: W \rightarrow W$ has a fixed point $\hat{u}$ i.e. $f(\hat{u})=\hat{u}$.

\section{Proof of Theorem 1}

By definition put

$$
W\left(T_{*}\right)=\left\{u \in E\left(T_{*}\right) \mid\|u\|_{\tau, \nu} \leq R, \quad 0<\tau<T_{*}, \quad 0<\nu<1\right\} .
$$

The constant $T_{*}>0$ will be defined.

First we find a fixed point of a mapping

$$
F(u)=\int_{0}^{t} S^{t-\xi} f(\xi, u(\xi)) d \xi
$$

This fixed point is the generalized solution announced in the second part of the theorem. Then by using formula (2.5) we show that this fixed point is the desired solution to problem (2.6).

Lemma 3. If the constat $T_{*}$ is small enough then the mapping $F$ takes the set $W\left(T_{*}\right)$ to itself.

Proof. Let constants $t, s$ be taken as follows $0<s<t^{1 / \gamma}, \quad t \leq T_{*}$. Suppose $u \in W\left(T_{*}\right)$ then estimate a function $v(t)=F(u)$ :

$$
\|v(t)\|_{s}^{E} \leq \int_{0}^{t}\left\|S^{t-\xi} f(\xi, u(\xi))\right\|_{s}^{E} d \xi=X+Y
$$


here we use the notation

$$
X=\int_{0}^{t-s^{\gamma}}\left\|S^{t-\xi} f(\xi, u(\xi))\right\|_{s}^{E} d \xi, \quad Y=\int_{t-s^{\gamma}}^{t}\left\|S^{t-\xi} f(\xi, u(\xi))\right\|_{s}^{E} d \xi .
$$

To estimate $X$ take constants $\varepsilon$ and $\mu$ such that

$$
0<\varepsilon<\frac{s}{t^{1 / \gamma}}<\mu<1 .
$$

The constant $\varepsilon$ is assumed to be small and the constant $\mu$ is assumed to be close to 1 .

Let the variables $\delta$ and $\delta^{\prime}$ be given by the formulas

$$
\delta=s-\varepsilon \xi^{1 / \gamma}, \quad \delta^{\prime}=\xi^{1 / \gamma}(\mu-\varepsilon) .
$$

Taking into account that $\xi \in\left(0, t-s^{\gamma}\right]$ we see that the variables $\delta, \delta^{\prime}$ are positive and

$$
s-\delta>0, \quad s-\delta+\delta^{\prime}<\xi^{1 / \gamma}, \quad \delta<(t-\xi)^{1 / \gamma} .
$$

The inequality in the middle implies that

$$
u(\xi) \in \bar{B}_{s-\delta+\delta^{\prime}}(R)
$$

and thus the term $X$ is estimated as follows

$$
\begin{aligned}
X & \leq C \int_{0}^{t-s^{\gamma}}(t-\xi)^{-\phi}\|f(\xi, u(\xi))\|_{s-\delta}^{G} d \xi \leq C^{2} \int_{0}^{t-s^{\gamma}} \frac{1}{\delta^{\alpha \alpha}(t-\xi)^{\phi}} d \xi \\
& \leq\left.\frac{C^{2}}{(\mu-\varepsilon)^{\alpha}} \int_{0}^{t-s^{\gamma}} \frac{d \xi}{(t-\xi)^{\phi} \xi^{\alpha / \gamma}}\right|_{\xi=y t}=\frac{C^{2} t^{1-\chi}}{(\mu-\varepsilon)^{\alpha}} \int_{0}^{1-s^{\gamma} / t} \frac{d y}{(1-y)^{\phi} y^{\alpha / \gamma}} \\
& \leq \frac{C^{2} J t^{1-\chi}}{(\mu-\varepsilon)^{\alpha}}, \quad J=\int_{0}^{1} \frac{d y}{(1-y)^{\phi} y^{\alpha / \gamma}}
\end{aligned}
$$

We shall estimate the term $Y$.

Introduce a function $\psi$ by the formula

$$
\psi(y)=y^{1 / \gamma}+(1-y)^{1 / \gamma}-1 .
$$

The function $\psi$ is positive on the interval $(0,1)$. Define a constant $I$ as follows

$$
I=\int_{0}^{1} \frac{d y}{(1-y)^{\phi}(\psi(y))^{\alpha}} .
$$

Let the constant $\mu$ be as above. We redefine the variables $\delta, \delta^{\prime}$ by the formulas

$$
\delta=\mu(t-\xi)^{1 / \gamma}, \quad \delta^{\prime}=\mu \xi^{1 / \gamma}+\delta-s .
$$

Now the variable $\xi$ belongs to the interval $\left[t-s^{\gamma}, t\right]$ and thus the variables $\delta, \delta^{\prime}$ are positive and satisfy inequalities (4.3).

It is only not trivial to show that the variable $\delta^{\prime}$ is positive. Let us prove this. Indeed,

$$
\delta^{\prime}=\mu \xi^{1 / \gamma}+\mu(t-\xi)^{1 / \gamma}-s=t^{1 / \gamma}\left(\mu y^{1 / \gamma}+\mu(1-y)^{1 / \gamma}-\frac{s}{t^{1 / \gamma}}\right),
$$


recall that $y=\xi / t$. Form (4.6) it follows that

$$
\delta^{\prime}>t^{1 / \gamma} \mu \psi(y)
$$

By the same argument as above, inclusion (4.4) is fulfilled with the new $\delta$ and $\delta^{\prime}$.

We are ready to estimate the term $Y$. By (4.7) it follows that

$$
\begin{aligned}
Y & \leq C \int_{t-s^{\gamma}}^{t}(t-\xi)^{-\phi}\|f(\xi, u(\xi))\|_{s-\delta}^{G} d \xi \leq C^{2} \int_{t-s^{\gamma}}^{t} \frac{d \xi}{(t-\xi)^{\phi} \delta^{\prime \alpha}} \\
& \leq \frac{C^{2} t^{1-\chi}}{\mu^{\alpha}} \int_{1-s^{\gamma} / t}^{1} \frac{d y}{(1-y)^{\phi}(\psi(y))^{\alpha}} \leq \frac{C^{2} I}{\mu^{\alpha}} t^{1-\chi} .
\end{aligned}
$$

Now the assertion the of lemma follows from formulas (4.1), (4.5) and (4.8).

Corollary 1. Formulas (4.5), (4.8) imply that if $0<s^{\gamma}<t \leq T_{*}$ and $v(t)=F(u), \quad u \in W\left(T_{*}\right)$ then

$$
\|v(t)\|_{s}^{E} \leq c_{2} t^{1-\chi}
$$

here $c_{2}$ is a positive constant independent on $u, t, s$.

Lemma 4. The set $F\left(W\left(T_{*}\right)\right)$ is precompact in $E\left(T_{*}\right)$.

Proof. By proposition 1 it is sufficient to prove that the set $F\left(W\left(T_{*}\right)\right)$ is uniformly continuous.

Take a function $u \in W\left(T_{*}\right)$ and let $v(t)=F(u)$. We must show that if $t^{\prime}, t^{\prime \prime} \geq \tau, \quad \tau \in\left(0, T_{*}\right)$ then for any $\mu \in(0,1)$ one has

$$
\sup _{u \in W\left(T_{*}\right)}\left\|v\left(t^{\prime}\right)-v\left(t^{\prime \prime}\right)\right\|_{\mu \tau^{1 / \gamma}}^{E} \rightarrow 0, \quad \text { as } \quad\left|t^{\prime}-t^{\prime \prime}\right| \rightarrow 0 .
$$

Indeed, for definiteness assume that $t^{\prime \prime}>t^{\prime}$ then

$$
\begin{aligned}
v\left(t^{\prime \prime}\right)-v\left(t^{\prime}\right) & =\int_{t^{\prime}}^{t^{\prime \prime}} S^{t^{\prime \prime}-\xi} f(\xi, u) d \xi \\
& +\left(S^{t^{\prime \prime}-t^{\prime}}-\operatorname{id}_{E_{s}}\right) \int_{0}^{t^{\prime}} S^{t^{\prime}-\xi} f(\xi, u) d \xi, \quad s^{\gamma}<\tau .
\end{aligned}
$$

Choose a positive constant $\delta$ such that $(s+\delta)^{\gamma}<\tau$ and using the parabolicity of the semigroup $S^{t}$ estimate the first term from the right side of this formula

$$
\begin{aligned}
\left\|\int_{t^{\prime}}^{t^{\prime \prime}} S^{t^{\prime \prime}-\xi} f(\xi, u) d \xi\right\|_{s}^{E} & \leq C \int_{t^{\prime}}^{t^{\prime \prime}}\left(t^{\prime \prime}-\xi\right)^{-\phi}\|f(\xi, u)\|_{s}^{G} d \xi \\
& \leq C^{2} \int_{t^{\prime}}^{t^{\prime \prime}} \frac{d \xi}{\delta^{\alpha}\left(t^{\prime \prime}-\xi\right)^{\phi}}=\frac{C^{2}}{\delta^{\alpha}(1-\phi)}\left(t^{\prime \prime}-t^{\prime}\right)^{1-\phi} .
\end{aligned}
$$

So that the first term in the right side of (4.9) is vanished uniformly.

Consider a set

$$
U=\bigcup_{\tau \leq t^{\prime} \leq T_{*}}\left\{\int_{0}^{t^{\prime}} S^{t^{\prime}-\xi} f(\xi, u) d \xi \mid u \in W\left(T_{*}\right)\right\} .
$$


By lemma 3 the set $U$ is bounded in any space $E_{\mu^{\prime} \tau^{1 / \gamma}}$ with $1>\mu^{\prime}>\mu$ thus it is compact in $E_{\mu \tau^{1 / \gamma}}$. By lemma 2 we get

$$
\sup _{w \in U}\left\|S^{t^{\prime \prime}-t^{\prime}} w-w\right\|_{\mu \tau^{1 / \gamma}}^{E} \rightarrow 0, \quad \text { as } \quad t^{\prime \prime}-t^{\prime} \rightarrow 0 .
$$

This shows that the second term in the right side of formula (4.9) is vanished uniformly.

Corollary 2. The set $F\left(W\left(T_{*}\right)\right)$ is uniformly continuous with respect to the variable $t$.

Lemma 5. The mapping $F: W\left(T_{*}\right) \rightarrow W\left(T_{*}\right)$ is continuous with respect to the topology of the space $E\left(T_{*}\right)$.

Proof. Suppose a sequence $\left\{v_{l}\right\} \subset W\left(T_{*}\right)$ to be convergent to the element $v \in W\left(T_{*}\right)$ as $l \rightarrow \infty$. We need to show that for any $s^{\gamma}<\tau<T_{*}$ the sequence

$$
\sup _{\tau \leq t \leq T_{*}}\left\|\int_{0}^{t} S^{t-\xi} f\left(\xi, v_{l}(\xi)\right) d \xi-\int_{0}^{t} S^{t-\xi} f(\xi, v(\xi)) d \xi\right\|_{s}^{E}
$$

vanishes as $l \rightarrow \infty$.

By corollary 2 the sequence

$$
\left\{\int_{0}^{t} S^{t-\xi} f\left(\xi, v_{l}(\xi)\right) d \xi\right\}
$$

is uniformly continuous on the interval $\left[\tau, T_{*}\right]$. The uniform convergence of such a sequence is equivalent to its pointwise convergence [10]. Thus it is sufficient to prove that sequence (4.10) is convergent in $E_{s}$ for each $t \in\left[\tau, T_{*}\right]$.

Fix $t \in\left[\tau, T_{*}\right]$ and let constants $\varepsilon, \mu$ satisfy inequality (4.2). Then using the argument of lemma 3 write

$$
\begin{aligned}
& \left\|\int_{0}^{t} S^{t-\xi}\left(f\left(\xi, v_{l}(\xi)\right)-f(\xi, v(\xi))\right) d \xi\right\|_{s}^{E} \\
& \quad \leq \int_{0}^{t-s^{\gamma}}(t-\xi)^{-\phi}\left\|f\left(\xi, v_{l}(\xi)\right)-f(\xi, v(\xi))\right\|_{\varepsilon \xi^{1 / \gamma}}^{G} d \xi \\
& \quad+\int_{t-s^{\gamma}}^{t}(t-\xi)^{-\phi}\left\|f\left(\xi, v_{l}(\xi)\right)-f(\xi, v(\xi))\right\|_{s-\mu(t-\xi)^{1 / \gamma}}^{G} d \xi .
\end{aligned}
$$

Since the function $f$ is continuous, for a fixed $\xi$ we have:

$$
\begin{aligned}
(t-\xi)^{-\phi}\left\|f\left(\xi, v_{l}(\xi)\right)-f(\xi, v(\xi))\right\|_{\varepsilon \xi \xi^{1 / \gamma}}^{G} \rightarrow 0, & \xi \in\left[0, t-s^{\gamma}\right], \\
(t-\xi)^{-\phi}\left\|f\left(\xi, v_{l}(\xi)\right)-f(\xi, v(\xi))\right\|_{s-\mu(t-\xi)^{1 / \gamma}}^{G} \rightarrow 0, & \xi \in\left[t-s^{\gamma}, t\right),
\end{aligned}
$$

as $l \rightarrow \infty$. 
Moreover by formulas (4.5), (4.8) both of these expressions are majorized with the $L^{1}$-integrable function:

$$
\begin{aligned}
& (t-\xi)^{-\phi}\left\|f\left(\xi, v_{l}(\xi)\right)-f(\xi, v(\xi))\right\|_{\varepsilon \xi^{1 / \gamma}}^{G} \\
& \quad \leq(t-\xi)^{-\phi}\left(\left\|f\left(\xi, v_{l}(\xi)\right)\right\|_{\varepsilon \xi^{1 / \gamma}}^{G}+\|f(\xi, v(\xi))\|_{\varepsilon \xi^{1 / \gamma}}^{G}\right) \leq \frac{2 C^{2}}{(\mu-\varepsilon)^{\alpha} \xi^{\alpha / \gamma}(t-\xi)^{\phi}},
\end{aligned}
$$

and

$$
(t-\xi)^{-\phi}\left\|f\left(\xi, v_{l}(\xi)\right)-f(\xi, v(\xi))\right\|_{s-\mu(t-\xi)^{1 / \gamma}}^{G} \leq \frac{2 C^{2}}{t^{\alpha / \gamma} \mu^{\alpha}(\psi(\xi / t))^{\alpha}(t-\xi)^{\phi}} .
$$

Therefore by the Dominated convergence theorem the integrals in the right side of (4.11) are vanished as $l \rightarrow \infty$.

So by theorem 3 and lemmas 3, 4, 5 we obtain a fixed point of the mapping $F$, say $u$ :

$$
F(u)=u \in W\left(T_{*}\right) .
$$

This proves the second part of theorem 1 .

To prove the first one let us show that this fixed point is the solution to problem (2.6). Suppose that $t, t+h>s^{\gamma}$. First consider the case $h>0$. Differentiate the function $u(t)$ explicitly:

$$
\begin{aligned}
u_{t}(t) & =\lim _{h \rightarrow 0} h^{-1}\left(\int_{0}^{t+h} e^{A(t+h-\xi)} f(\xi, u(\xi)) d \xi-\int_{0}^{t} e^{A(t-\xi)} f(\xi, u(\xi)) d \xi\right) \\
& =\lim _{h \rightarrow 0} h^{-1} \int_{t}^{t+h} e^{A(t+h-\xi)} f(\xi, u(\xi)) d \xi \\
& +\lim _{h \rightarrow 0} h^{-1}\left(e^{A h}-\operatorname{id}_{E_{s}}\right) \int_{0}^{t} e^{A(t-\xi)} f(\xi, u(\xi)) d \xi
\end{aligned}
$$

Lemma 3 implies that $\int_{0}^{t} e^{A(t-\xi)} f(\xi, u(\xi)) d \xi \in E_{s^{\prime}}$ with $s^{\gamma}<s^{\prime \gamma}<t, t+h$ hence formula (2.5) gives

$$
h^{-1}\left(e^{A h}-\operatorname{id}_{E_{s}}\right) \int_{0}^{t} e^{A(t-\xi)} f(\xi, u(\xi)) d \xi \rightarrow A \int_{0}^{t} e^{A(t-\xi)} f(\xi, u(\xi)) d \xi
$$

in $E_{s}$ as $h \rightarrow 0$.

Let us prove that

$$
h^{-1} \int_{t}^{t+h} e^{A(t+h-\xi)} f(\xi, u(\xi)) d \xi \rightarrow f(t, u(t))
$$

in $E_{s}$ as $h \rightarrow 0$. 
Indeed, observe that

$$
\begin{aligned}
h^{-1} & \int_{t}^{t+h} e^{A(t+h-\xi)} f(\xi, u(\xi)) d \xi-f(t, u(t)) \\
& =h^{-1}\left(\int_{t}^{t+h} e^{A(t+h-\xi)}(f(\xi, u(\xi))-f(t, u(t))) d \xi\right. \\
& \left.+\int_{t}^{t+h}\left(e^{A(t+h-\xi)}-\operatorname{id}_{E_{s}}\right) f(t, u(t)) d \xi\right) .
\end{aligned}
$$

The first integral in the right side of this formula is estimated as follows:

$$
\begin{aligned}
& \left\|\int_{t}^{t+h} e^{A(t+h-\xi)}(f(\xi, u(\xi))-f(t, u(t))) d \xi\right\|_{s}^{E} \\
& \quad \leq C h \max _{t \leq \xi \leq t+h}\|f(\xi, u(\xi))-f(t, u(t))\|_{s}^{E}=o(h) .
\end{aligned}
$$

Since the semigroup $e^{A t}$ is strongly continuous for the second integral we get

$$
\begin{aligned}
& \left\|\int_{t}^{t+h}\left(e^{A(t+h-\xi)}-\mathrm{id}_{E_{s}}\right) f(t, u(t)) d \xi\right\|_{s}^{E} \\
& \quad \leq h \max _{t \leq \xi \leq t+h}\left\|\left(e^{A(t+h-\xi)}-\mathrm{id}_{E_{s}}\right) f(t, u(t))\right\|_{s}^{E}=o(h) .
\end{aligned}
$$

If $h<0$ then instead of formula (4.12) one must use the following expression

$$
\begin{aligned}
u_{t}(t) & =\lim _{h \rightarrow 0} h^{-1}\left(\left(\operatorname{id}_{E_{s}}-e^{-A h}\right) \int_{0}^{t+h} e^{A(t+h-\xi)} f(\xi, u(\xi)) d \xi\right. \\
& \left.-\int_{t+h}^{t} e^{A(t-\xi)} f(\xi, u(\xi)) d \xi\right) .
\end{aligned}
$$

In this case only the proof of the formula

$$
\begin{aligned}
\lim _{h \rightarrow 0} h^{-1}\left(\operatorname{id}_{E_{s}}\right. & \left.-e^{-A h}\right) \int_{0}^{t+h} e^{A(t+h-\xi)} f(\xi, u(\xi)) d \xi \\
& =A \int_{0}^{t} e^{(t-\xi)} f(\xi, u(\xi)) d \xi
\end{aligned}
$$

differs from the previous argument.

Let us prove this formula. Obviously we have

$$
\begin{aligned}
\left(\operatorname{id}_{E_{s}}\right. & \left.-e^{-A h}\right) \int_{0}^{t+h} e^{A(t+h-\xi)} f(\xi, u(\xi)) d \xi \\
& =\left(\operatorname{id}_{E_{s}}-e^{-A h}\right) u(t)+\left(\operatorname{id}_{E_{s}}-e^{-A h}\right)(u(t+h)-u(t)) .
\end{aligned}
$$

The set

$$
V=\left\{\frac{u(t+h)-u(t)}{\|u(t+h)-u(t)\|_{s^{\prime}}^{E}} \mid h \in\left(h^{\prime}, 0\right)\right\}
$$


with $h^{\prime}<0$ close to zero is bounded in $E_{s^{\prime}}, \quad s^{\gamma}<s^{\prime \gamma}<t+h^{\prime}$. Consequently $V$ is a compact set in $E_{s}$. By lemma 2 the set

$$
\left(A_{-h}-A\right) V, \quad A_{-h}=\frac{1}{h}\left(\operatorname{id}_{E_{s}}-e^{-A h}\right)
$$

is bounded in $E_{s}$ and thus the set $A_{-h} V$ is also bounded.

Thus taking into account that the function $u(t)$ is continuous we yield

$$
\begin{aligned}
\left\|\frac{1}{h}\left(\operatorname{id}_{E_{s}}-e^{-A h}\right)(u(t+h)-u(t))\right\|_{s}^{E} & \\
& =\|u(t+h)-u(t)\|_{s^{\prime}}^{E} \cdot\left\|A_{-h} \frac{u(t+h)-u(t)}{\|u(t+h)-u(t)\|_{s^{\prime}}^{E}}\right\|_{s}^{E}=o(1) .
\end{aligned}
$$

For the second term of the right side of (4.15) this implies

$$
\left\|\left(\operatorname{id}_{E_{s}}-e^{-A h}\right)(u(t+h)-u(t))\right\|_{s}^{E}=o(h) .
$$

The first term of the right side of formula (4.15) is estimated as follows

$$
\left\|\left(\operatorname{id}_{E_{s}}-e^{-A h}\right) u(t)-h A u(t)\right\|_{s}^{E}=o(h) .
$$

Substituting formulas (4.13) and (4.14) to (4.12) we see that the function $u$ is a solution to equation (2.6).

Formula (2.9) follows from corollary 1.

Theorem 1 is proved.

\section{Applications}

In the sequel we denote all the inessential positive constants by the same letter $c$.

5.1. Parabolic equation with gradient nonlinearity. In this section we consider a model example.

Let $M \subset \mathbb{R}^{m}$ be a bounded domain with smooth boundary $\partial M$.

Consider the following equation

$$
u_{t}=f(\nabla u)+\Delta u,\left.\quad u\right|_{t=0}=\hat{u} \in H_{0}^{1, q}(M), \quad u(t, \partial M)=0, \quad t>0,
$$

here $q>1$.

The function $f$ is almost everywhere continuous in $\mathbb{R}^{m}$ and for almost all $z \in \mathbb{R}^{m}$ we have $|f(z)| \leq c\left(|z|^{p}+1\right), \quad q \geq p \geq 1$. Note that the function $f$ may not necessarily be a Lipschitz function.

Let us show that if

$$
m(p-1)<q .
$$

then problem (5.1) has a generalized solution from $C\left([0, T], H_{0}^{1, q}(M)\right)$, the constant $T>0$ depends on $\hat{u}$.

If the function $f$ is a Lipschitz function then inequality (5.2) is well known: it corresponds to the subcritical case in the sense of Fujita.

After the change of the unknown function $u=e^{\Delta t} \hat{u}+v$ our problem takes the form

$$
v_{t}=g(t, x, \nabla v)+\Delta v,\left.\quad v\right|_{t=0}=0, \quad g(t, x, \nabla v)=f\left(\nabla\left(e^{\Delta t} \hat{u}+v\right)\right) .
$$


Consider problem (5.3) in the scales

$$
E_{s}=H_{0}^{1+s_{0}+s, q}(M), \quad\|\cdot\|_{s}=\|\cdot\|_{H_{0}^{1+s_{0}+s, q}(M)}, \quad s \in(0, S),
$$

and

$$
G_{s}=H^{-\lambda, q}(M), \quad\|\cdot\|^{G}=\|\cdot\|_{H^{-\lambda, q}(M)},
$$

this means that all the spaces $G_{s}$ coincide with each other, the constants $S>0, s_{0} \geq 0$ and $0 \leq \lambda<m(1-1 / q)$ to be defined.

Introduce a constant

$$
r=\frac{q m}{m+\lambda q} \in(1, q]
$$

Then using standard facts on the Sobolev spaces estimate the function $g$ :

$$
\begin{aligned}
\|g(t, x, \nabla v)\|^{G} & \leq c\|g(t, x, \nabla v)\|_{L^{r}(M)} \leq c\left(\left\|\nabla\left(e^{\Delta t} \hat{u}+v\right)\right\|_{L^{p r}(M)}^{p}+1\right) \\
& \leq c\left(\left\|e^{\Delta t} \hat{u}\right\|_{H^{1, p r}(M)}^{p}+\|v\|_{H^{1, p r}(M)}^{p}+1\right) .
\end{aligned}
$$

Choose a constant $s_{0}$ as follows

$$
s_{0}=m\left(\frac{1}{q}-\frac{1}{r p}\right) .
$$

Then the condition $H_{0}^{1+s_{0}, q}(M) \subseteq H_{0}^{1, p r}(M)$ is satisfied.

Here we assume that the constant $\lambda$ is such that we have $q<r p$. Note that

$$
\left\|e^{\Delta t} \hat{u}\right\|_{H^{1, p r}(M)}^{p} \leq c t^{-\beta}\|\hat{u}\|_{H^{1, q}(M)}^{p}, \quad \beta=\frac{m}{2}\left(\frac{p}{q}-\frac{1}{r}\right) .
$$

If $v \in B_{s}=\left\{h \in E_{s} \mid\|h\|_{s}^{E} \leq 1\right\}$ then by all these argument formula (5.4) implies

$$
\|g(t, x, \nabla v)\|^{G} \leq \frac{c}{t^{\beta}} .
$$

Another inequality we need is

$$
\left\|e^{\Delta t} w\right\|_{s} \leq c t^{-\phi}\|w\|^{G}, \quad \phi=\frac{1+s_{0}+s+\lambda}{2},
$$

this formula also follows from the standard Sobolev spaces theory.

Proposition 2. The mapping $(t, v) \mapsto g(t, x, \nabla v)$ is a continuous mapping of $(0, T) \times B_{s}$ to $G_{s}$.

Proof. Assume the converse: there exists a sequence $\left(t_{k}, v_{k}\right)$ such that $t_{k} \rightarrow$ $t \in(0, T), \quad v_{k} \rightarrow v$ in $E_{s}$ as $k \rightarrow \infty, \quad v, v_{k} \in B_{s}$ and

$$
\left\|g_{k}(x)-g(x)\right\|^{G} \geq c>0
$$

here we put $g_{k}(x)=g\left(t_{k}, x, \nabla v_{k}\right), \quad g(x)=g(t, x, \nabla v)$.

By the argument above formula (5.5) imply

$$
\left\|g_{k}(x)-g(x)\right\|_{L^{r}(M)} \geq c>0,
$$

Since $\nabla v_{k} \rightarrow \nabla v$ in $L^{p r}(M)$ then there exists a subsequence $\left\{v_{k^{\prime}}\right\} \subseteq\left\{v_{k}\right\}$ such that $\nabla v_{k^{\prime}} \rightarrow \nabla v$ almost every where in $M$. Thus $\left|g_{k^{\prime}}(x)-g(x)\right|^{r} \rightarrow 0$ almost everywhere in $M$. Consequently $\left|g_{k^{\prime}}(x)-g(x)\right|^{r} \rightarrow 0$ in measure. 
It remains to show that the sequence $\left|g_{k^{\prime}}(x)-g(x)\right|^{r}$ is uniformly integrable. If we do this then by the Vitali convergence theorem [6] it follows that $\left\|g_{k^{\prime}}(x)-g(x)\right\|_{L^{r}(M)} \rightarrow 0$ and this contradiction proves the Proposition.

Note that since $v_{k}, v \in E_{s} s>0$ we actually have $\nabla v_{k} \rightarrow \nabla v$, in $L^{p r+\sigma}(M)$ with small $\sigma>0$. Thus the functions $g_{k^{\prime}}(x)-g(x)$ belong not only to $L^{r}(M)$ but also to $L^{r+\varepsilon}(M)$ with small $\varepsilon>0$ and the sequence $\left\|g_{k^{\prime}}(x)-g(x)\right\|_{L^{r+\varepsilon}(M)}$ is bounded (these observations follow from the same argument as above). The last observation can be rewritten as follows:

$$
\begin{gathered}
\sup _{k^{\prime}} \int_{M}\left|g_{k^{\prime}}(x)-g(x)\right|^{r} æ\left(\left|g_{k^{\prime}}(x)-g(x)\right|\right) d x \\
=\sup _{k^{\prime}}\left\|g_{k^{\prime}}(x)-g(x)\right\|_{L^{r+\varepsilon}(M)}^{r+\varepsilon}<\infty
\end{gathered}
$$

with $æ(y)=y^{\varepsilon}$. Since the function $x$ is monotone and unbounded in $\mathbb{R}_{+}$, this proves the uniform integrability of the sequence $\left|g_{k^{\prime}}(x)-g(x)\right|^{r}$.

Now we see that $\alpha=0$ and to apply theorem 1 we need $\chi=\phi+\beta<1$. It is easy to show that the last inequality follows from (5.2) if only the constant $S$ is sufficiently small and the constant $\lambda$ is chosen to make the expression $p r$ to be sufficiently close to $q$.

5.2. The scale of analytic functions. Let $\mathbb{T}^{m}=\mathbb{R}^{m} /(2 \pi \mathbb{Z})^{m}$ be the $m$-dimensional torus. All the technique developed below can be transferred almost literally to the case of the problem with zero boundary conditions on the $m$-dimensional cube.

By $x=\left(x_{1}, \ldots, x_{m}\right)$ denote an element of $\mathbb{R}^{m}$.

Let $\mathbb{T}_{s}^{m}=\left\{z=x+i y \in \mathbb{C}^{m}\left|x \in \mathbb{T}^{m}, \quad\right| y_{j} \mid<s, \quad j=1, \ldots, m\right\}$ be the complex neighborhood of the torus $\mathbb{T}^{m}$.

Define a set $E_{s}, \quad s>0$ as follows $E_{s}=C\left(\overline{\mathbb{T}}_{s}^{m}\right) \bigcap \mathcal{O}\left(\mathbb{T}_{s}^{m}\right)$. Here $\mathcal{O}\left(\mathbb{T}_{s}^{m}\right)$ stands for the set of analytic functions in $\mathbb{T}_{s}^{m}$.

The set $E_{s}$ is a Banach space with respect to the norm $\|u\|_{s}=\max _{z \in \overline{\mathbb{T}}_{s}^{m}}|u(z)|$. By the Montel theorem the embeddings $E_{s+\delta} \subset E_{s}, \quad \delta>0$ are completely continuous. By definition put $E_{0}=C\left(\mathbb{T}^{m}\right)$ and $\|\cdot\|_{0}=\|\cdot\|_{C\left(\mathbb{T}^{m}\right)}$.

Let $\Delta$ stands for the standard Laplace operator

$$
\Delta=\sum_{j=1}^{m} \partial_{j}^{2}, \quad \partial_{j}=\frac{\partial}{\partial x_{j}}
$$

Lemma 6. There exists a positive constant c such that for any $u \in E_{s}, \quad s \geq$ 0 the following inequality holds

$$
\left\|e^{t \Delta} u\right\|_{s+\delta} \leq c \exp \left(\frac{\delta^{2}}{4 t}\right)\|u\|_{s}, \quad t, \delta>0 .
$$

The constant $c$ depends only on $m$. 
Proof. The assertion of the lemma easily follows from the well-known formula:

$$
\left(e^{t \Delta} u\right)(x)=\frac{1}{(4 \pi t)^{m / 2}} \int_{\mathbb{R}} e^{-\left(\xi_{1}-x_{1}\right)^{2} /(4 t)} d \xi_{1} \ldots \int_{\mathbb{R}} e^{-\left(\xi_{m}-x_{m}\right)^{2} /(4 t)} d \xi_{m} u(\xi) .
$$

In all these integrals one must shift the contour of integration to the complex plane and then the desired inequality follows from the standard estimates.

By lemma 6 the semigroup $e^{t \Delta}$ is parabolic with $\gamma=2$.

Lemma 7. Take a constant $\rho \in(0,1 / 2]$. For any $\varepsilon \in(0,2 \rho)$ there is a positive constant $c=c(\varepsilon)$ such that if $u \in E_{s+\delta}$ then

$$
\begin{aligned}
\left\|(-\Delta)^{-\rho} \partial_{j} u\right\|_{s} & \leq \frac{c}{\delta^{1-2 \rho+\varepsilon}}\|u\|_{s+\delta}, \quad s \geq 0, \quad \delta>0, \\
\left\|(-\Delta)^{\rho} u\right\|_{s} & \leq \frac{c}{\delta^{2 \rho+\varepsilon}}\|u\|_{s+\delta} .
\end{aligned}
$$

Proof. Let us prove formula (5.7). Using the standard facts on Sobolev's spaces we have

$$
\left\|(-\Delta)^{-\rho} \partial_{j} u\right\|_{s} \leq c\left\|(-\Delta)^{-\rho} \partial_{j} u\right\|_{H^{\varepsilon, p}\left(\mathbb{T}_{s}^{m}\right)} \leq c\|u\|_{H^{\varepsilon+1-2 \rho, p}\left(\mathbb{T}_{s}^{m}\right)}, \quad \varepsilon p>2 m .
$$

Then the desired result follows from the interpolation formula and the Cauchy inequality:

$$
\|u\|_{H^{\varepsilon+1-2 \rho, p}\left(\mathbb{T}_{s}^{m}\right)} \leq c\|u\|_{H^{1, p}\left(\mathbb{T}_{s}^{m}\right)}^{\varepsilon+1-2 \rho}\|u\|_{L^{p}\left(\mathbb{T}_{s}^{m}\right)}^{2 \rho-\varepsilon}, \quad\|u\|_{H^{1, p}\left(\mathbb{T}_{s}^{m}\right)} \leq \frac{c}{\delta}\|u\|_{s+\delta} .
$$

Formula (5.8) is derived in the same way.

Proposition 3 ([11]). For any constants $a \geq r \geq 0$ one has

$$
\left\|e^{t \Delta} u\right\|_{H^{a}\left(\mathbb{T}^{m}\right)} \leq \frac{c}{t^{(a-r) / 2}}\|u\|_{H^{r}\left(\mathbb{T}^{m}\right)} .
$$

If $a>m / 2$ then $\|u\|_{0} \leq c\|u\|_{H^{a}\left(\mathbb{T}^{m}\right)}$.

The first of the following two examples illustrates the effect described in the Introduction, the second one is to compare our result with the known one.

5.3. Integro-differential parabolic equation. In this section we use the scales $G_{s}=E_{s}=C\left(\overline{\mathbb{T}}_{s}^{m}\right) \bigcap \mathcal{O}\left(\mathbb{T}_{s}^{m}\right)$. Let us focus our attention on a one dimensional $(m=1)$ system.

Consider a problem

$u_{t}=\left\|\left.(-\Delta)^{n} u\right|_{\mathbb{T}^{m}}\right\|_{L^{2}(\mathbb{T})}^{\lambda}+\Delta u,\left.\quad u\right|_{t=0}=\hat{u}(x)=\sum_{|k| \geq 2} \frac{e^{i k x}}{|k|^{1 / 2} \log |k|} \in L^{2}(\mathbb{T})$.

Here $\lambda$ is a positive parameter, $n \in \mathbb{N}$.

Parabolic equations with right side depending on $L^{p}$ norms of the unknown function arise in the theory of incompressible viscous fluid [9]. 
After the change of variable $u=e^{t \Delta} \hat{u}+v$ our problem takes the form

$$
v_{t}=f(t, v)+\Delta v,\left.\quad v\right|_{t=0}=0, \quad f(t, v)=\left\|(-\Delta)^{n} e^{t \Delta} \hat{u}+(-\Delta)^{n} v\right\|_{L^{2}(\mathbb{T})}^{\lambda} .
$$

One can show that $f$ is a Lipschitz function:

$$
\left|f\left(t, v^{\prime}\right)-f\left(t, v^{\prime \prime}\right)\right| \leq \frac{c}{t^{n} s^{2 n}}\left\|v^{\prime}-v^{\prime \prime}\right\|_{s}
$$

But this property can not save the situation: the denominator $t^{n} s^{2 n}$ is too small to find a solution by means of a successive procedure. So it is convenient to ignore this Lipschitz inequality and write more effective estimates.

Let us show that if $n \lambda<1$ then problem (5.9) has a solution in the sense of theorem 1.

So that one has $|f(t, v)| \leq c\left(\left\|e^{t \Delta} \hat{u}\right\|_{H^{2 n}(\mathbb{T})}^{\lambda}+\left\|(-\Delta)^{n} v\right\|_{L^{2}(\mathbb{T})}^{\lambda}\right)$. Then using proposition 3 we obtain $\left\|e^{t \Delta} \hat{u}\right\|_{H^{2 n}(\mathbb{T})} \leq c t^{-n}\|\hat{u}\|_{L^{2}(\mathbb{T})}$. The Cauchy inequality gives

$$
\left\|(-\Delta)^{n} v\right\|_{L^{2}(\mathbb{T})} \leq c\left\|(-\Delta)^{n} v\right\|_{s} \leq c \delta^{-2 n}\|v\|_{s+\delta}, \quad \delta>0 .
$$

Combining these inequalities with each other and taking into account that $(s+\delta)^{2}<t$ we have

$$
|f(t, v)| \leq c \delta^{-2 n \lambda}\left(\|\hat{u}\|_{L^{2}(\mathbb{T})}^{\lambda}+\|v\|_{s+\delta}^{\lambda}\right) .
$$

Thus $\chi=n \lambda$ and if $n \lambda<1$ then by theorem 1 the problem has at least one analytic solution.

Consider the case $\lambda=1$ and let for simplicity $n=1$.

Denote by $u_{k}$ the Fourier coefficients of a function $u: u(x)=\sum_{k \in \mathbb{Z}} u_{k} e^{i k x}$. Notice that the norm of $L^{2}(\mathbb{T})$ can be presented as follows

$$
\|u\|_{L^{2}(\mathbb{T})}^{2}=c \sum_{k \in \mathbb{Z}}\left|u_{k}\right|^{2}
$$

Then separating the variables in problem (5.9) we obtain

$$
\begin{aligned}
& u_{0}=c \int_{0}^{t}\left(\sum_{|k| \geq 2} \frac{|k|^{3} e^{-2 \xi|k|^{2}}}{(\log |k|)^{2}}\right)^{\frac{1}{2}} d \xi, \\
& u_{k}=0, \quad \text { if } \quad|k|=1, \\
& u_{k}=\frac{e^{-t|k|^{2}}}{|k|^{1 / 2} \log |k|}, \quad \text { if } \quad|k| \geq 2 .
\end{aligned}
$$

It is not difficult to show that

$$
\left(\sum_{|k| \geq 2} \frac{|k|^{3} e^{-2 \xi|k|^{2}}}{(\log |k|)^{2}}\right)^{\frac{1}{2}} \geq-\frac{c}{\xi \log \xi}, \quad \xi \in(0,1) .
$$

So that the integral in formula (5.11) does not exist and thus there are no solutions in this case. 
5.4. 3-D Navier-Stokes equation. In this section we use the scale $G_{s}=$ $E_{s}=C\left(\overline{\mathbb{T}}_{s}^{m}\right) \bigcap \mathcal{O}\left(\mathbb{T}_{s}^{m}\right)$.

Consider the Navier-Stokes equation in the divergence free setup. After Leray's projection the Navier-Stokes equation takes the well-known form

$$
\begin{aligned}
\left(u^{k}\right)_{t} & =A_{l}^{k} \partial_{j}\left(u^{j} u^{l}\right)+\Delta u^{k}, \quad A_{l}^{k}=\left(\Delta^{-1} \partial_{k} \partial_{l}-\delta_{k l}\right), \\
\left.u^{k}\right|_{t=0} & =\hat{u}^{k} \in H^{r}\left(\mathbb{T}^{3}\right),
\end{aligned}
$$

where $\delta_{k l}=1$ for $k=l$ and 0 otherwise; $k, l, j=1,2,3$ we also use the Einstein summation convention.

From [8], [7] it follows that if $r=1 / 2$ then problem (5.12) has a solution $u^{i}(t, x)$ which is regular in the spatial variables for all $t \in\left(0, T_{*}\right)$. Here $T_{*}$ is a small positive constant.

Let us show that by theorem 1 the analytic solution exists for all $r>1 / 2$. This indicates that in terms of paper [1] theorem 1 allows us to carry out only the subcritical case. This is no surprise since theorem 1 is very general.

Assume a parameter $\rho \in(0,1 / 2)$ to be close $1 / 2$ and let us change the variable in (5.12): $u^{k}=e^{t \Delta} \hat{u}^{k}+(-\Delta)^{\rho} v^{k}$. Then the problem has the form

$$
v_{t}^{k}=f^{k}(t, v)+\Delta v^{k},\left.\quad v^{k}\right|_{t=0}=0,
$$

here

$$
\begin{aligned}
f^{k}(t, v) & =A_{l}^{k} \partial_{j}(-\Delta)^{-\rho}\left(e^{t \Delta} \hat{u}^{j} e^{t \Delta} \hat{u}^{l}+e^{t \Delta} \hat{u}^{j}(-\Delta)^{\rho} v^{l}\right. \\
& \left.+(-\Delta)^{\rho} v^{j} e^{t \Delta} \hat{u}^{l}+(-\Delta)^{\rho} v^{j}(-\Delta)^{\rho} v^{l}\right) .
\end{aligned}
$$

Estimate the function $f$ term by term. Using lemma 7 we have

$$
\begin{aligned}
\left\|A_{l}^{k} \partial_{j}(-\Delta)^{-\rho}\left((-\Delta)^{\rho} v^{j}(-\Delta)^{\rho} v^{l}\right)\right\|_{s} \leq \frac{c}{\delta^{\varepsilon+1-2 \rho}} \sum_{j, l=1}^{3}\left\|(-\Delta)^{\rho} v^{j}(-\Delta)^{\rho} v^{l}\right\|_{s+\delta / 2} \\
\quad \leq \frac{c}{\delta^{\varepsilon+1-2 \rho}} \sum_{j, l=1}^{3}\left\|(-\Delta)^{\rho} v^{j}\right\|_{s+\delta / 2}\left\|(-\Delta)^{\rho} v^{l}\right\|_{s+\delta / 2} \\
\leq \frac{c}{\delta^{\varepsilon+1+2 \rho}} \sum_{j, l=1}^{3}\left\|v^{j}\right\|_{s+\delta}\left\|v^{l}\right\|_{s+\delta} .
\end{aligned}
$$

Now one must choose the parameters $\varepsilon>0, \quad \rho \in(0,1 / 2)$ such that

$$
\frac{\varepsilon+1+2 \rho}{2}<1
$$


Let us estimate another term of the function $f$ by using lemmas6, 7 and proposition $3\left((s+\delta)^{2}<t\right)$ :

$$
\begin{aligned}
\left\|A_{l}^{k} \partial_{j}(-\Delta)^{-\rho}\left(e^{t \Delta} \hat{u}^{j} e^{t \Delta} \hat{u}^{l}\right)\right\|_{s} & \leq \frac{c}{\delta^{1+\varepsilon-2 \rho}} \sum_{j, l=1}^{3}\left\|e^{t \Delta} \hat{u}^{j}\right\|_{s+\delta}\left\|e^{t \Delta} \hat{u}^{l}\right\|_{s+\delta} \\
& \leq \frac{c}{\delta^{1+\varepsilon-2 \rho}} \sum_{j, l=1}^{3}\left\|e^{t \Delta / 2} \hat{u}^{j}\right\|_{0}\left\|e^{t \Delta / 2} \hat{u}^{l}\right\|_{0} \\
& \leq \frac{c}{\delta^{1+\varepsilon-2 \rho}} \sum_{j, l=1}^{3}\left\|e^{t \Delta / 2} \hat{u}^{j}\right\|_{H^{a}\left(\mathbb{T}^{3}\right)}\left\|e^{t \Delta / 2} \hat{u}^{l}\right\|_{H^{a}\left(\mathbb{T}^{3}\right)} \\
& \leq \frac{c}{\delta^{1+\varepsilon-2 \rho} t^{a-r}} \sum_{j, l=1}^{3}\left\|\hat{u}^{j}\right\|_{H^{r}\left(\mathbb{T}^{3}\right)}\left\|\hat{u}^{l}\right\|_{H^{r}\left(\mathbb{T}^{3}\right)},
\end{aligned}
$$

here $a>3 / 2$. We need to have

$$
\frac{1+\varepsilon-2 \rho}{2}+a-r<1 .
$$

In the same manner we obtain

$$
\left\|A_{l}^{k} \partial_{j}(-\Delta)^{-\rho}\left(e^{t \Delta} \hat{u}^{j}(-\Delta)^{\rho} v^{l}\right)\right\|_{s} \leq \frac{c}{\delta^{\varepsilon+1} t^{(a-r) / 2}} \sum_{j, l=1}^{3}\left\|\hat{u}^{j}\right\|_{H^{r}\left(\mathbb{T}^{3}\right)}\left\|v^{l}\right\|_{s+\delta} .
$$

Thus there must be

$$
\varepsilon+1+a-r<2 .
$$

It is not difficult to show that for any $r>1 / 2$ there exists the small parameter $\varepsilon>0$, the parameter $a$ close to $3 / 2$ from above and the parameter $\rho$ close to $1 / 2$ from below such that inequalities $(5.13),(5.14),(5.15)$ are fulfilled.

\section{REFERENCES}

[1] J. M. Arrieta A. N. Carvalho Abstract parabolic problems with critical nonlinearities and applications to Navier-Stokes and Heat equations. Trans. of the Amer. Math. Soc. V. 352, 1, pp. 285-310.

[2] F. E. Browder A new generalization of the Schauder fixed point theorem, Math. Ann. 174, (1967), 285-290.

[3] A. N. Carvalho Abstract parabolic problems in odered Banach spaces. Cadernos de Mathematica 02, 141-146, March (2001) Artigo Numero SMA No.104.

[4] J. Diedonné Deux exeples singuliers d'équations différentielles, Acta. Scien. Math. (Szeged) 12 (1950), B 38-40.

[5] A. Godunov Peano's theorem in Banach spaces, Functional Anal. Appl. 9 (1975), 53-55.

[6] G. Folland. Real Analysis: Modern Techniques and Their Applications, second ed. Wiley-Interscience, 1999.

[7] H. Fujita, T. Kato On the Navier-Stokes initial value problem I. Arch. Rat. Mech. Anal. 16 (1964), 269-315. MR 29:3774.

[8] T. Kato, H. Fujita On the nonstationary Navier-Stokes system. Rend. Sem. Math. Univ. Padova 32, (1962), 243-260. MR 26:495. 
[9] K. Ohkitani and H. Okamoto, Blow-up problems modeled from the strain-vorticity dynamics, Proceedings of "Tosio Kato's Method and Principles for Evolution Equations in Mathematical Physics" ( Eds. H. Fujita, S. T. Kuroda and H. Okamoto), RIMS Kokyuroku 1234 (2001), pp. 240-250.

[10] L. Schwartz Analyse Mathématique, Hermann, 1967.

[11] M. E. Taylor Partial Differential Equations, Springer, New York, 1996.

[12] J. Yorke A continuous differential equation in Hilbert space without existence, Funkcialaj Ekvacioj 13 (1970), 19-21.

[13] O. Zubelevich On Some Topological View on the Abstract Cauchy-Kowalewski Problem. Complex Variables, August 15, 2004, vol. 49, no. 10, pp. 703-709(7).

E-mail address: ozubel@yandex.ru

Current address: 2-nd Krestovskii Pereulok 12-179, 129110, Moscow, Russia 